\title{
Torreya nucifera seed oil improves 3T3-L1 adipocyte differentiation
}

\author{
Eunbi Koh, Boram Kim and Kyungoh Choi ${ }^{*}$ (1)
}

\begin{abstract}
Background: Adipose tissue is a critical regulator of lipid storage and endocrine function. Impairment of the recruitment of new adipocytes in the adipose tissue is associated with ectopic fat accumulation, diabetes and insulin resistance. Torreya nucifera, an evergreen conifer that grows in warm temperate climates, has been found to exert beneficial effects against inflammation, infection and diabetes. However, the molecular mechanisms responsible for these effects at the cellular level remain unknown. This study aimed to investigate effects of Torreya nucifera seed oil (TNSO) on 3T3-L1 adipocyte differentiation and its underlying regulatory mechanism.

Methods: To investigate the effects of TNSO on adipocyte differentiation, 3T3-L1 cells were induced to differentiate for 5 days in the presence of $0.75 \mu \mathrm{L} / \mathrm{mL}$ TNSO. Oil Red $O$ staining and an assay for intracellular triglyceride were performed to determine the extent of lipid accumulation in 3T3-L1 cells. To elucidate the underlying mechanism of TNSO, adipogenic gene expression was analyzed using quantitative real-time PCR. Moreover, we monitored TNSOderived activation of PPARY and STAT3 with 3T3-L1 reporter cell lines engineered to secrete Gaussia luciferase upon the interaction of a transcription factor to its DNA binding element.

Results: Oil Red O staining revealed that TNSO improved the differentiation of 3T3-L1 preadipocytes into mature adipocytes. The mRNA levels of adipogenic genes, including adiponectin, fatty acid synthase (FAS) and adipocyte fatty acid-binding protein (FABP4), were upregulated and intracellular triglyceride levels increased upon TNSO treatment. We also established that adipocyte differentiation was improved by TNSO-derived activation of PPARY and STAT3.
\end{abstract}

Conclusions: Our results suggest that TNSO improves adipocyte differentiation by regulating the activation of adipogenic transcription factors, indicating that it may serve as a potential treatment strategy for adipocyte dysfunction.

Keywords: Torreya nucifera seed oil, Adipocyte, Differentiation, PPARY, STAT3

\section{Background}

Adipose tissue regulates energy storage and energy supply in the body. Healthy adipose tissue relies on a steady renewal of adipocytes, which differentiate in a multistep process called adipogenesis. Adipogenesis is crucial for metabolic homeostasis and impairment of adipogenesis is a prominent characteristic of obesity and insulin resistance [1]. In particular, during aging, physiological

\footnotetext{
*Correspondence: kyungoh98@suwon.ac.kr
}

Department of Chemical and Material Engineering, The University

of Suwon, 17, Wauan-gil, Bongdam-eup, Hwaseong-si, Gyeonggi-do

18323, Republic of Korea reactive oxygen species (ROS) levels are altered and conventional adipogenesis is disrupted. The suppression of adipocyte renewal via oxidative stress increases the risk of developing hypertrophic adipocytes, lipotoxicity and insulin resistance [2].

Adipocyte differentiation is a complex process involving several genes, whose expression is regulated through various adipocyte-specific transcription factors $[3,4]$. A key transcription factor in the adipocyte differentiation program is peroxisome proliferator-activated receptor gamma (PPAR $\gamma$ ) and signal transducer and activator of transcription 3 (STAT3) also plays a critical role in the regulation of other adipocyte-specific 
transcription factors [5, 6]. The degree of activation of these transcription factors is associated with the expression of adipocyte-specific genes including fatty acid synthase (FAS), adipocyte fatty acid-binding protein (FABP4) and adiponectin, which regulate metabolic processes in adipocytes [7-9].

Torreya nucifera is an evergreen conifer growing in Korea, China, and Southern Japan. According to previous reports, Torreya nucifera has been widely used as a traditional medicine owing to its beneficial effects on helminth infestation, constipation, diabetes mellitus, and hemorrhoids [10, 11]. Previous studies on Torreya nucifera primarily focused on the physicochemical properties of extracts from its leaves, fruits, and wood. Yoon et al. showed that essential oils drived from Torreya nucifera leaves suppressed growth of skin pathogens and secretion of inflammatory substances by macrophage cells [12]. An ethyl acetate fraction prepared from the seed of Torreya nucifera exhibited antiinflammatory activities in macrophage cells [11]. In addition, the ethanol extract of Torreya nucifera leaves exhibited inhibitory activity on the main protease of coronavirus that causes severe acute respiratory syndrome [13]. Although Torreya nucifera has been widely used traditionally, its effects on adipocyte differentiation and the underlying mechanism at the cellular level have not previously been studied.

In this study, we investigated the effects of Torreya nucifera seed oil (TNSO) on 3T3-L1 adipocyte differentiation. Adipocyte differentiation was assessed using Oil Red O staining, measurement of intracellular triglyceride levels, and quantitative real-time PCR which was performed to analyze adipogenic gene expression. Furthermore, to elucidate the mechanism underlying the effects of TNSO, a change of PPARY and STAT3 activation was monitored using 3T3-L1 reporter cell lines.

\section{Methods \\ Chemicals}

Torreya nucifera seed oil (TNSO) was obtained from Durae Corporation (Gunpo, Korea). Bovine serum (BS) was purchased from Gibco (Grand Island, NY, USA). Fetal bovine serum (FBS) and Dulbecco's modified Eagle's medium (DMEM) were purchased from Hyclone (Logan, UT, USA). Penicillin, streptomycin and phosphatebuffered saline (PBS) were purchased from Capricorn (Ebsdorfergrund, Hesse, Germany). Dimethyl sulfoxide (DMSO), insulin, dexamethasone (DEX), 3-iso-butyl1-methylxanthine (IBMX), 3,3',5-Triiodo-L-thyronine (T3) and the other chemicals were purchased from Sigma-Aldrich (St. Louis, MO, USA).

\section{Cell culture and differentiation}

Murine 3T3-L1 cells were provided by Prof. Barbara Corkey (Boston University School of Medicine, MA, USA). 3T3-L1 preadipocytes were seeded into 12-well plates at a density of $5 \times 10^{4}$ cells $/ \mathrm{mL}$ and cultured in growth media containing DMEM supplemented with BS $(10 \%$ $\mathrm{v} / \mathrm{v})$, streptomycin $(10 \mathrm{mg} / \mathrm{mL})$ and penicillin $(10,000 \mathrm{U} /$ $\mathrm{mL})$ at $37^{\circ} \mathrm{C}$ in a humidified atmosphere of $5 \% \mathrm{CO}_{2}$. Two days after the cells approached $100 \%$ confluency (day 0 ), differentiation was induced with an adipogenic cocktail $(1 \mu \mathrm{M}$ DEX, $0.5 \mathrm{mM}$ IBMX, $1 \mu \mathrm{g} / \mathrm{mL}$ insulin, and $2 \mathrm{nMT} 3)$ added to basal medium (DMEM with FBS (10\% $\mathrm{v} / \mathrm{v}$ ) and penicillin/streptomycin). On day 2 , the first differentiation medium was replaced with the second differentiation medium consisting of the basal medium supplemented with $2 \mathrm{nMT} 3$ and $1 \mu \mathrm{g} / \mathrm{mL}$ insulin. On day 4 , the cells were further differentiated with the basal medium. TNSO was dissolved in DMSO and diluted with the culture medium to obtain the desired concentrations. TNSO was added on days 0,2 , and 4 , while the control group was treated only with DMSO. The final concentration of DMSO was equal between the TNSO-treated and control groups.

\section{Measurement of cell viability}

The viability of cultured cells was determined via the methylthiazolyldiphenyl tetrazolium bromide (MTT) method. Cells were cultured in 24-well plates at a density of $2.5 \times 10^{4}$ cells $/ \mathrm{mL}$. After $24 \mathrm{~h}$ of TNSO treatment, $50 \mu \mathrm{L}$ of $5 \mathrm{mg} / \mathrm{mL}$ MTT solution was added to each well and the cells were incubated for $4 \mathrm{~h}$ at $37^{\circ} \mathrm{C}$. Thereafter, $500 \mu \mathrm{L}$ of DMSO was added to dissolve the formazan crystals. After $15 \mathrm{~min}$, absorbance was measured at $590 \mathrm{~nm}$ using a microplate spectrophotometer (BioTek Instruments, Winooski, VT, USA).

\section{Oil Red O staining}

On day 5, post-differentiation induction, 3T3-L1 cells were washed with PBS, fixed with $10 \%$ formalin solution for $20 \mathrm{~min}$, and washed with $60 \%$ isopropyl alcohol. After drying completely, the cells were stained with $0.5 \%$ Oil Red O solution for $1 \mathrm{~h}$ at room temperature. Thereafter, stained cells were washed four times with distilled water, and photomicrographs were obtained using an Eclipse Ti2 inverted microscope (Nikon, Tokyo, Japan).

\section{Measurement of intracellular triglyceride levels}

On day 5, post-differentiation induction, the medium was removed and 3T3-L1 cells were washed once with PBS. The cells were disrupted using lysis buffer $(0.1 \%$ SDS, $100 \mathrm{mM}$ Tris- $\mathrm{HCl}$, and $1 \mathrm{mM}$ EDTA) and the freezethaw cycle between $-80^{\circ} \mathrm{C}$ and $37^{\circ} \mathrm{C}$ was repeated three 
times. The intracellular triglyceride content was measured using a triglyceride determination kit (Sigma, St. Louis, MO, USA) in accordance with the manufacturer's instructions. The absorbance was measured at $540 \mathrm{~nm}$ using a microplate spectrophotometer (BioTek Instruments, Winooski, VT, USA).

\section{Quantitative real-time PCR (qRT-PCR) analysis}

To examine the effect of TNSO on adipogenic gene expression, 3T3-L1 preadipocytes were allowed to differentiate for 5 days with $0.75 \mu \mathrm{L} / \mathrm{mL}$ TNSO. TNSO was added on days 0,2 , and 4 , whereas the control group was treated only with DMSO. On day 5 , post-differentiation induction, total RNA of 3T3-L1 cells was extracted using the RNeasy Mini Kit (QIAGEN, Hilden, Germany). Target RNA was amplified using the AccuPower ${ }^{\circledR}$ GreenStar $^{\mathrm{TM}}$ RT-qPCR Master Mix (Bioneer, Daejeon, Korea). Quantitative RT-PCR was performed on LightCycler ${ }^{\circledR}$ 96 system (Roche, Basel, Switzerland) as follows: reverse transcription at $60^{\circ} \mathrm{C}$ for $15 \mathrm{~min}$, pre-denaturation at $95^{\circ} \mathrm{C}$ for $5 \mathrm{~min}$, denaturation at $95^{\circ} \mathrm{C}$ for $10 \mathrm{~s}$, annealing at $55^{\circ} \mathrm{C}$ for $30 \mathrm{~s}$, and extension at $72^{\circ} \mathrm{C}$ for $30 \mathrm{~s}$. The denaturation step to extension step was cycled 45 times. Relative mRNA expression levels were normalized to those of $\beta$-actin as the internal control. The sequences of the primers were as follows: $5^{\prime}$-GAGGTATCCTGACCCTGA AGTA-3' ( $\beta$-actin forward), 5'-CACACGCAGCT CAT TGTAGA-3 ${ }^{\prime}$ ( $\beta$-actin reverse), $5^{\prime}$-TGGAAGACAGCT CCTCCTCG-3' (FABP4 forward), 5'-AATCCCCATTTA CG CTGATGATC-3' (FABP4 reverse), 5'-ACCTGGTAG ACCACTGCATTGAC-3' (FAS forward), 5'-CCTGAT GAAACGACACATTCTCA-3' (FAS reverse), 5'-TGA GACAG GAGATGTTGGAATG-3' (adiponectin forward), 5'-ACGCTGAGCGATACACATA AG-3' (adiponectin reverse).

\section{Measurement of PPAR $\gamma$ and STAT3 activation using Gaussia luciferase (GLuc) reporter cells}

Stable 3T3-L1 PPARY and STAT3 reporter cell lines were generated as follows. Briefly, consensus binding sites of PPAR $\gamma$ and STAT3 (AGGACAAAGGTCA for PPAR $\gamma$ and TTTCCGGGAA for STAT3) were identified using the TRANSFAC public database. The response element (RE) oligonucleotides, containing three consensus binding sequences, were cloned into the GLuc-DRE2-viral vector, wherein GLuc is regulated by a minimal promoter [14]. The expression of GLuc is induced when a target transcription factor binds to its consensus binding site. Thereafter, stable 3T3-L1 PPAR $\gamma$ and STAT3 reporter cell lines were generated through lentiviral transduction.

To profile the activation of PPAR $\gamma$ and STAT3, the reporter cells were differentiated in 12-well plates as described above. The supernatant was harvested on day 1 , day 3 , and day 5 post-differentiation induction and used to measure GLuc activity. GLuc activity (Relative Light Units; RLU) was assessed using the Gaussia Luciferase Flash Assay Kit (Thermo Fisher Scientific, Waltham, MA, USA) and TriStar ${ }^{2}$ LB 942 Modular Multimode Microplate Reader (Berthold technologies, Bad Wildbad, Germany) in accordance with the manufacturers' instructions. After normalization with cell density, relative fold changes were determined at each time point.

\section{Statistical analysis}

All tests were performed with two sets of triplicate experiments. Statistical comparisons between samples were performed using Student's $t$-test.

\section{Results}

TNSO does not affect 3T3-L1 viability

To determine the viability of 3T3-L1 cells upon treatment with TNSO, cells were incubated with various concentrations $(0.1-0.75 \mu \mathrm{L} / \mathrm{mL})$ of TNSO. As shown in Fig. 1, TNSO displayed no toxicity towards 3T3-L1 cells at all tested concentrations. Additionally, the potential toxic effect for another cell line was examined and TNSO treatment did not decrease the viability of RAW 264.7 cells (Supplementary Fig. 1).

\section{TNSO increases lipid accumulation during 3T3-L1 adipocyte differentiation}

To examine the effect of TNSO on 3T3-L1 adipocyte differentiation, 3T3-L1 preadipocytes were allowed to differentiate for 5 days with $0.75 \mu \mathrm{L} / \mathrm{mL}$ TNSO. As shown by Oil Red O staining, TNSO treatment increased the number of stained lipid droplets (Fig. 2a). For quantitative comparison, we further performed a triglyceride determination assay to measure intracellular triglyceride levels. As a result, the triglyceride content of TNSO-treated cells was approximately 8.5 -fold higher than that of the control group (Fig. 2b). These results suggest that TNSO treatment increases lipid accumulation, indicating the progression of adipocyte differentiation.

\section{TNSO upregulates adipogenic gene expression}

To further investigate the effect of TNSO on 3T3-L1 adipocyte differentiation, we analyzed the mRNA expression levels of adipogenic genes including adiponectin, FABP4, and FAS on day 5 post-differentiation induction. Adiponectin, a specific adipokine produced by adipocytes, was upregulated by 2.6 -fold with TNSO treatment. Furthermore, the levels of FABP4 and FAS, which play key roles in fatty acid metabolism, were significantly upregulated by 3.1-fold and 2.1-fold, respectively (Fig. 3). These results indicate that TNSO improves 3T3-L1 


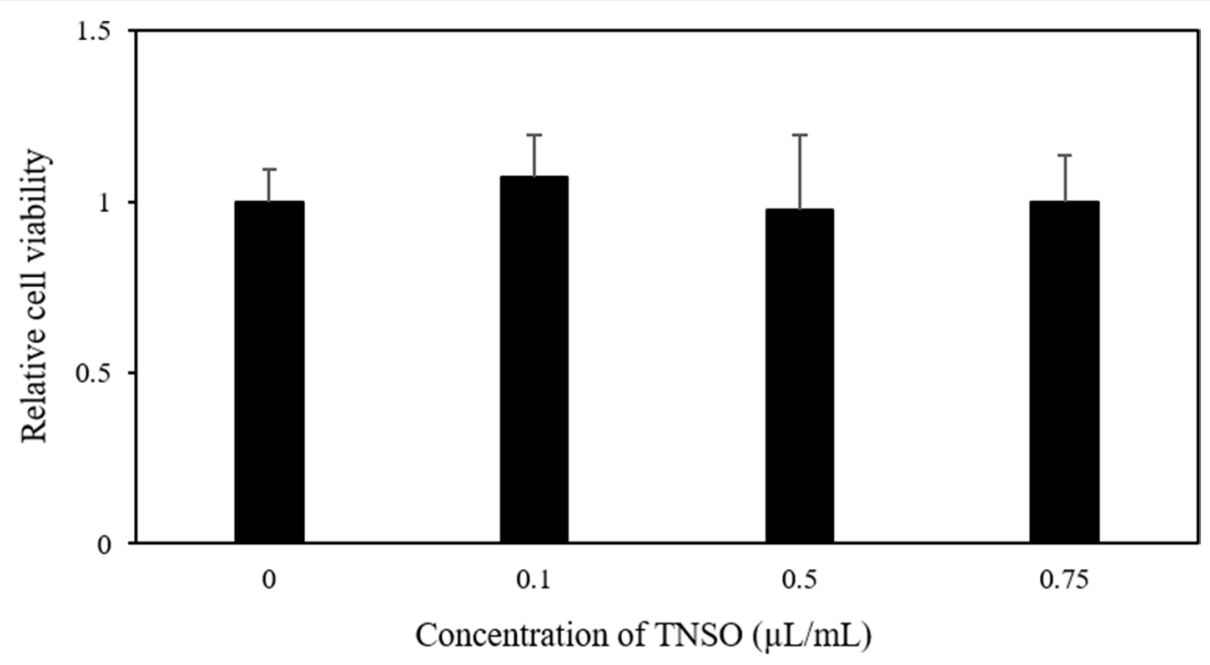

Fig. 1 The effect of Torreya nucifera seed oil (TNSO) on cell of 3T3-L1 cells. Cytotoxicity of 3T3-L1 cells treated with TNSO for 24h was determined using the methylthiazolyldiphenyl tetrazolium bromide assay. All tests were performed with two sets of triplicate experimental setups. Bars indicate mean and SEM

A
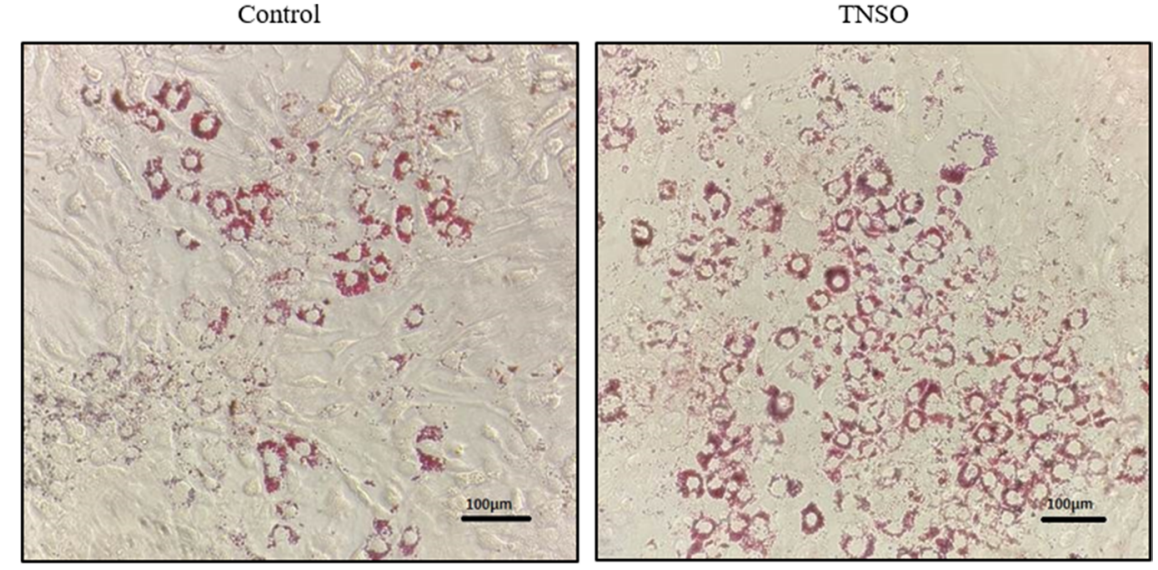

B

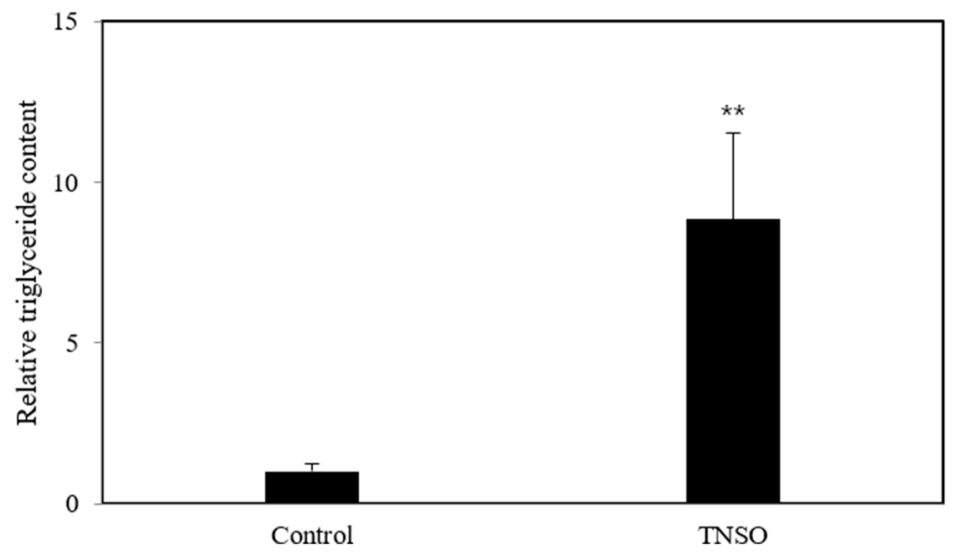

Fig. 2 The effect of Torreya nucifera seed oil (TNSO) on lipid droplet formation and intracellular triglyceride contents in 3T3-L1 cells. 3T3-L1 preadipocytes were cultured for 5 days post-differentiation induction in medium supplemented with DMSO or TNSO dissolved in DMSO. a Representative images of 3T3-L1 adipocytes stained with Oil Red O. Scale bar $=100 \mu \mathrm{m} \mathbf{b}$ Intracellular triglyceride contents in 3T3-L1 adipocytes. All tests were performed in triplicate in two independent experiments. The bars indicate mean and SEM. ${ }^{* *}$ : $p<0.01$ compared to the samples treated with DMSO 


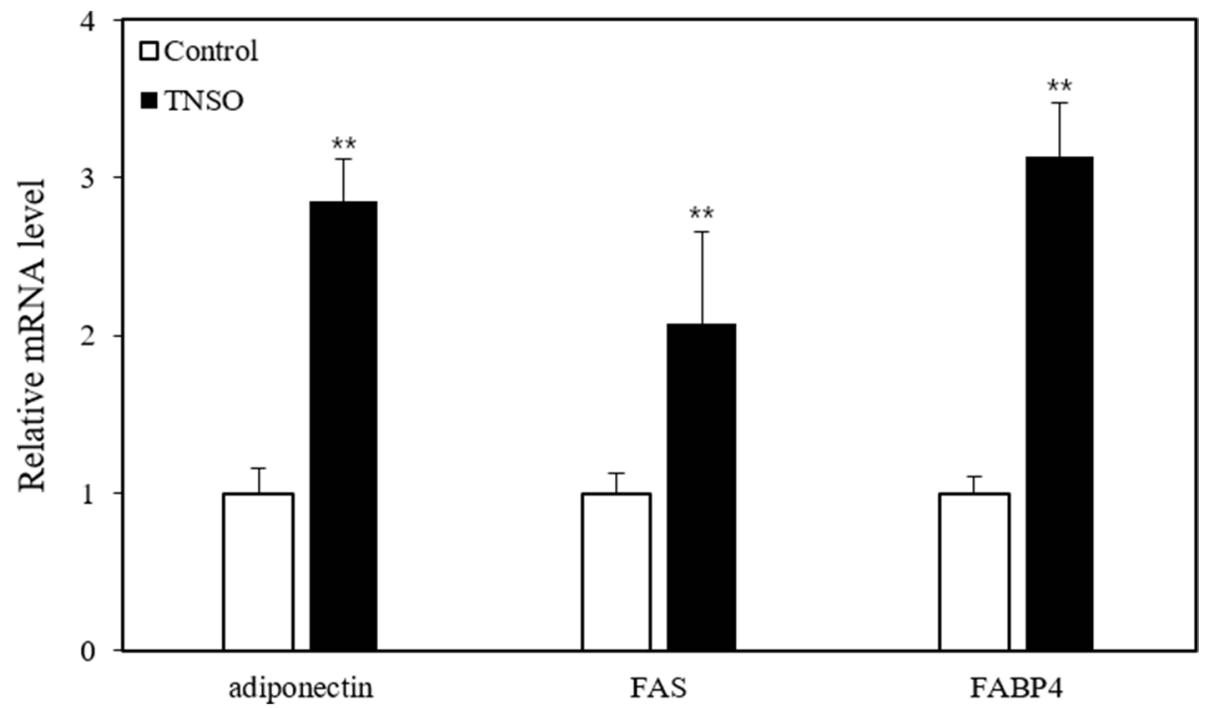

Fig. 3 The effect of Torreya nucifera seed oil (TNSO) on the expression of adipogenic genes. Total RNA was extracted from 3T3-L1 adipocytes on day 5 post-differentiation induction. The mRNA levels of adiponectin, fatty acid synthase (FAS) and adipocyte fatty acid-binding protein 2 (FABP4) were determined through quantitative real-time PCR analysis and normalized with corresponding $\beta$-actin mRNA expression levels. All tests were performed in triplicate in two independent experiments. The bars indicate mean and SEM. ${ }^{* *}: p<0.01$ compared to samples treated with DMSO

adipocyte differentiation by upregulating adipogenic gene expression.

\section{TNSO induces PPARY and STAT3 activation}

Adipocyte differentiation is a highly complex process involving morphological alteration and changes in the expression of several adipogenic genes [15]. Hence, it is necessary to investigate how TNSO affects the activation of transcription factors regulating the expression of adipogenic genes. Changes in transcription factor activity profiles were monitored using 3T3-L1 reporter cell lines, which report the activation of a target transcription factor through secreted Gaussia luciferase. PPARy functions as a master regulator of adipogenic genes and STAT3 is reported to be involved in the early stage of $3 \mathrm{~T} 3-\mathrm{L} 1$ adipocyte differentiation [16, 17]. In this study, $0.75 \mu \mathrm{L} / \mathrm{mL}$ TNSO treatment increased PPAR $\gamma$ activity by $44 \%$ on day 1 and a similar degree of increased activity was maintained until day 5 after the induction of differentiation (Fig. 4a). In the case of STAT3, a significant increase by $84 \%$ was detected on day 5 (Fig. 4b). These results suggest that TNSO-induced activation of PPAR $\gamma$ and STAT3 contributes to the improvement in adipocyte differentiation.

\section{Discussion}

TNSO has been used as an edible oil and traditional medicine. Although studies on Torreya nucifera have previously shown anti-bacterial, anti-inflammatory and anti-viral activities, limited information has been available regarding its effects on adipocyte metabolism at the cellular level. Hence, this study aimed to investigate the effects of TNSO on 3T3-L1 adipocyte differentiation and the underlying mechanism. We found that the formation of lipid droplets and intracellular triglyceride content were increased upon TNSO treatment (Fig. 2). To confirm the stimulatory role of TNSO on adipocyte differentiation, we compared the expression level of genes reflecting characteristics of adipocyte differentiation. Adiponectin is a specific adipokine produced by adipocytes and exerts anti-apoptotic, anti-inflammatory and insulin-sensitive effects $[9,18]$. FABP4 is a lipid-binding protein playing a key role in intracellular transport and metabolism of fatty acids $[8,19]$. FAS is an enzyme that mediates fatty acid synthesis [7, 20]. In this study, adiponectin, FABP4 and FAS levels were upregulated upon TNSO treatment during 3T3-L1 adipocyte differentiation (Fig. 3).

Adipocyte differentiation is regulated in a complicated manner through the interaction of several transcription factors. A previous study reported a marked increase in the activity of major adipogenic transcription factors at the early stages of the adipocyte differentiation process $[21,22]$. Hence, we monitored the activity of PPAR $\gamma$ and STAT3, which are closely associated with adipocyte differentiation, to elucidate the mechanism of action of TNSO. Consequently, PPARy activity peaked upon TNSO treatment on day 1 and remained approximately $30 \%$ higher than that in the control group until day 5 . Although STAT3 activity did not significantly change until day 3, it almost doubled on day 5 upon TNSO 

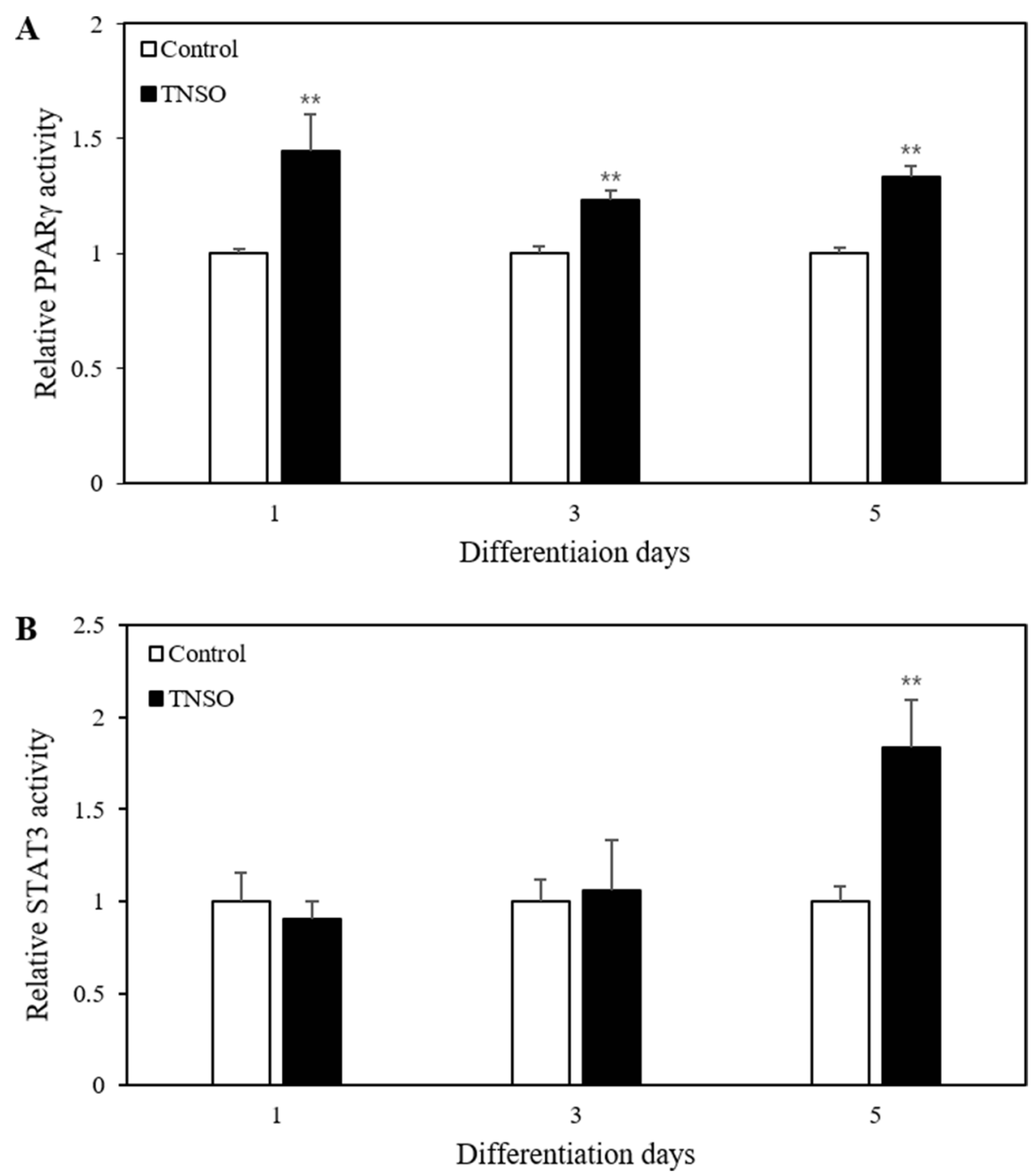

Fig. 4 The effect of Torreya nucifera seed oil (TNSO) on the activation of PPARY and STAT3. 3T3-L1 reporter cells for PPARY or STAT3 were differentiated and supernatants containing secreted Gaussia luciferase (GLuc) were harvested on days 1, 3, and 5 post-differentiation induction. Relative activity of $\mathbf{a}$ PPARY and $\mathbf{b}$ STAT3 was determined from the fold-change normalized with the activity of cells treated with DMSO at each time point. All tests were performed in triplicate in two independent experiments. The bars indicate mean and SEM. **: $p<0.01$ compared to samples treated with DMSO

treatment. Deng et al. reported that induction of differentiation with an adipogenic cocktail led to an increase of STAT3 activity [5]. As the differentiation-inducing reagent is removed from day 4, the difference in STAT3 activation by TNSO starts to appear from day 5. Wang et al. showed that STAT3 inhibition suppressed adipocyte differentiation, suggesting that STAT3 signaling occurs upstream of PPAR $\gamma$ [23]. Because PPAR $\gamma$ activity increased from day 1, TNSO-derived PPAR $\gamma$ activation appeared to be independent of STAT3 activation. Numerous studies have reported that the expression of adiponectin, FAS and FABP4 is regulated by STAT3 and
PPAR $[5,7,8]$. Thus, the enhancement of adipocyte differentiation upon TNSO treatment can be explained with the increase in adipogenic gene expression by PPAR $\gamma$ and STAT3 activation.

Impaired adipocyte differentiation is associated with aging, oxidative stress and insulin resistance. As the levels of oxidative stress and insulin resistance increase due to reduced adipocyte renewal during aging, a strategy for improvement of adipocyte differentiation is needed. The present results suggest that TNSO may serve as a potential agent to treat diseases resulting from suppressed adipocyte differentiation. 


\section{Conclusions}

Thus, this study shows that TNSO significantly improves 3T3-L1 adipocyte differentiation by modulating PPAR $\gamma$ and STAT3 activity, concomitantly upregulating adipogenic gene expression and increasing lipid accumulation in adipocytes. These results establish the pro-adipogenic effect of TNSO and provide insights into its underlying mechanism of action at the cellular level. Therefore, our data supports that TNSO may be a promising agent for the recovery of impaired adipogenesis.

\section{Abbreviations}

TNSO: Torreya nucifera seed oil; FAS: Fatty acid synthase; FABP4: Adipocyte fatty acid-binding protein; PPARY: Peroxisome proliferator-activated receptor gamma; STAT3: Signal transducer and activator of transcription 3.

\section{Supplementary Information}

The online version contains supplementary material available at https://doi. org/10.1186/s12906-021-03429-5.

Additional file 1: Supplementary Fig. 1. The effect of Torreya nucifera seed oil (TNSO) on viability of RAW264.7 cells

\section{Acknowledgements}

The authors thank the research deputy of The University of Suwon for financially supporting this study.

\section{Authors' contributions}

Conceived and designed the experiments: KC. Performed the experiments: EK BK. Analyzed the data: KC EK BK. Drafted the manuscript: KC EK BK. All authors have read and approved the final manuscript.

\section{Funding}

This study was supported by a research grant of The University of Suwon in 2018.

\section{Availability of data and materials}

The raw data generated during the current study are available from the corresponding author upon request.

\section{Declarations}

Ethics approval and consent to participate

Not applicable.

\section{Consent for publication}

Not applicable.

\section{Competing interests}

The authors declare no conflict of interest.

Received: 23 June 2021 Accepted: 29 September 2021

Published online: 07 October 2021

\section{References}

1. Ghaben AL, Scherer PE. Adipogenesis and metabolic health. Nat Rev Mol Cell Biol. 2019;20(4):242-58.
2. Castro JP, Grune T, Speckmann B. The two faces of reactive oxygen species (ROS) in adipocyte function and dysfunction. Biol Chem. 2016;397(8):709-24.

3. Rosen ED, Walkey CJ, Puigserver P, Spiegelman BM. Transcriptional regulation of adipogenesis. Genes Dev. 2000;14(11):1293-307.

4. Gregoire FM, Smas CM, Sul HS. Understanding adipocyte differentiation. Physiol Rev. 1998;78(3):783-809.

5. Deng J, Hua K, Lesser SS, Harp JB. Activation of signal transducer and activator of transcription-3 during proliferative phases of 3T3-L1 adipogenesis. Endocrinology. 2000;141(7):2370-6.

6. Lefterova MI, Zhang Y, Steger DJ, Schupp M, Schug J, Cristancho A, et al. PPARgamma and C/EBP factors orchestrate adipocyte biology via adjacent binding on a genome-wide scale. Genes Dev. 2008;22(21):2941-52.

7. Moustaïd N, Sul HS. Regulation of expression of the fatty acid synthase gene in 3T3-L1 cells by differentiation and triiodothyronine. J Biol Chem. 1991;266(28):18550-4.

8. Shan T, Liu W, Kuang S. Fatty acid binding protein 4 expression marks a population of adipocyte progenitors in white and brown adipose tissues. FASEB J. 2013;27(1):277-87.

9. Arita Y, Kihara S, Ouchi N, Takahashi M, Maeda K, Miyagawa J, et al. Paradoxical decrease of an adipose-specific protein, adiponectin, in obesity. Biochem Biophys Res Commun. 1999:257(1):79-83.

10. Chen SP, Dong M, Kita K, Shi QW, Cong B, Guo WZ, et al. Anti-proliferative and apoptosis-inducible activity of labdane and abietane diterpenoids from the pulp of Torreya nucifera in HeLa cells. Mol Med Rep. 2010;3(4):673-8.

11. Kim SH, Park JG, Hong YD, Kim E, Baik KS, Yoon DH, et al. Src/Syk/IRAK1targeted anti-inflammatory action of Torreya nucifera butanol fraction in lipopolysaccharide-activated RAW264.7 cells. J Ethnopharmacol. 2016;188:167-76.

12. Yoon W, Kim S-S, Oh T, Lee N, Hyun C. Torreya nucifera essential oil inhibits skin pathogen growth and lipopolysaccharide-induced inflammatory effects. Int J Pharmacol. 2009:5:37-43.

13. Ryu YB, Jeong HJ, Kim JH, Kim YM, Park JY, Kim D, et al. Biflavonoids from Torreya nucifera displaying SARS-CoV $3 \mathrm{CL}$ (pro) inhibition. Bioorg Med Chem. 2010;18(22):7940-7.

14. Tian J, Andreadis ST. Independent and high-level dual-gene expression in adult stem-progenitor cells from a single lentiviral vector. Gene Ther. 2009;16(7):874-84.

15. Fajas L, Fruchart JC, Auwerx J. Transcriptional control of adipogenesis. Curr Opin Cell Biol. 1998;10(2):165-73.

16. Zhang $\mathrm{K}$, Guo W, Yang Y, WU J. JAK2/STAT3 pathway is involved in the early stage of adipogenesis through regulating C/EBP $\beta$ transcription. J Cell Biochem. 2011:112(2):488-97.

17. Zhao P, Stephens JM. Identification of STAT target genes in adipocytes. Jakstat. 2013;2(2):e23092.

18. Zhu Q, An YA, Kim M, Zhang Z, Zhao S, Zhu Y, et al. Suppressing adipocyte inflammation promotes insulin resistance in mice. Mol Metab. 2020;39:101010.

19. Furuhashi M, Hotamisligil GS. Fatty acid-binding proteins: role in metabolic diseases and potential as drug targets. Nat Rev Drug Discov. 2008;7(6):489-503.

20. Kuhajda FP. Fatty acid synthase and cancer: new application of an old pathway. Cancer Res. 2006;66(12):5977-80.

21. Choi K, Ghaddar B, Moya C, Shi H, Sridharan GV, Lee K, et al. Analysis of transcription factor network underlying 3T3-L1 adipocyte differentiation. PLOS One. 2014:9(7):e100177.

22. Niemelä S, Miettinen S, Sarkanen J-R, Ashammakhi N. Adipose tissue and adipocyte differentiation: molecular and cellular aspects and tissue engineering applications. Top Tissue Eng. 2008;4(1):26.

23. Wang D, Zhou Y, Lei W, Zhang K, Shi J, Hu Y, et al. Signal transducer and activator of transcription 3 (STAT3) regulates adipocyte differentiation via peroxisome-proliferator-activated receptor gamma (PPARgamma). Biol Cell. 2009:102(1):1-12.

\section{Publisher's Note}

Springer Nature remains neutral with regard to jurisdictional claims in published maps and institutional affiliations. 